\title{
Biodegradation of Dodecyltrimethylammonium Bromide by Pseudomonas fluorescens F7 and F2 Isolated from Activated Sludge
}

\author{
NAOHIRO NISHIYAMA ${ }^{1 *}$ and TSUTOMU NISHIHARA ${ }^{2}$ \\ ${ }^{1}$ Safety and Environmental Research Center, Kao Corporation, 2606 Akabane, Ichikai-machi, Haga, Tochigi, \\ 321-3497, Japan \\ ${ }^{2}$ Graduate School of Pharmaceutical Sciences, Osaka University, 1-6 Yamada-oka, Suita, Osaka, 565-0871, \\ Japan
}

(Received April 25, 2002-Accepted July 23, 2002)

Two dodecyltrimethylammonium bromide (DTAB) — degrading bacteria were isolated from activated sludge of a wastewater treatment plant (WWTP). The bacteria identified as Pseudomonas fluorescens was called F2 and F7. The BOD biodegradability of DTAB by these bacteria were relatively high and similar to that of the activated sludge. F7 grew in a basal medium containing DTAB as the sole carbon source, producing trimethylamine (TMA) from DTAB by a N-dealkylation process. Then, the intermediate TMA was degraded to dimethylamine (DMA) by N-dimethylation as a second step. The pathway of the degradation of DTAB by this strain was the same as that of the activated sludge. On the other hand, Ps. fluorescens F2 degraded DTAB to TMA, but TMA was not further degraded. As for Ps. fluorescens F7 degrading activity, anionic compounds, such as fatty acids and sodium dodecyl sulfates (SDS), enhanced the degradation and dodecanoate became the carbon and energy source for this bacteria strain. These results demonstrate that monoalkyl quaternary ammonium compounds are ultimately degraded through $\mathrm{N}$-dealkylation and mineralization, by a bacterium isolated from activated sludge.

Key words: quaternary ammonium compounds, surfactant, biodegradation

Quaternary ammonium compounds (QACs), major surfactants, have been widely used as disinfectants, dispersion agents and other products. QACs have one or two hydrophobic alkyl chains at a quaternary nitrogen atom. When QACs are discharged into the aquatic environment, attention should be paid to their effects, because such chemicals have an antimicrobial action at relatively low concentrations (Kakiichi, 1993, Stephans et al., 1994, Kikuchi et al., 1996). A study on the biodegradation of QACs showed they were ultimately biodegradable when present at low concentrations in river surface water and/or sediments (Larson and Vashon. 1983). However, their biodegradation mechanism is little known, although there are some reports on monoalkyl or dialkyl-QACs degrading bacteria.

\footnotetext{
* Corresponding author; E-mail: nishiyama.naohiro@kao.co.jp, Tel: +81-285-68-7437; Fax: +81-285-68-7452
}

Dean-Raymond and Alexander (1977) reported that a mixture of Pseudomonas and Xanthomonas isolated from soil and sewage grew in a medium consisting of decyltrimethylammonium salt as a sole carbon source, and that Xanthomonas alone in the presence of yeast extract and casamino acids oxidized the terminal carbon of the long alkyl chain of the salt. van Ginkel (1992) demonstrated the cleavage of the N-C bond of hexadecylammonium salt by Pseudomonas sp. Nishiyama et al. (1995) showed the biodegradation pathway of monoalkyl-QACs by activated sludge obtained from a WWTP (domestic sewage mainly). Recently, Nishihara et al. (2000) found the biodegradation pathway of didodecyldimethylammonium salt and broad assimilation activity for QACs using a degrading bacteria isolated from activated sludge of a WWTP. Thus, it is worthwhile studying the microbial contribution to the degradation of monoalkyl-QACs. In this paper, we report the biodegrada- 
tion pathway of DTAB - degrading bacteria isolated from activated sludge from a WWTP.

\section{Materials and Methods}

\section{Isolation of bacteria and degradation assay}

DTAB - degrading bacteria were isolated from the activated sludge of a municipal wastewater treatment plant by using enrichment and agar plate cultures. The sludge was collected from a WWTP in Utsunomiya, Tochigi, Japan in March 1993. The basal medium used for enrichment culture consists of $15 \mathrm{mg}$ of DTAB, $30 \mathrm{mg}$ of activated sludge, 65.3 $\mathrm{mg}$ of $\mathrm{K}_{2} \mathrm{HPO}_{4}, 26.0 \mathrm{mg}$ of $\mathrm{KH}_{2} \mathrm{PO}_{4}, 134 \mathrm{mg}$ of $\mathrm{Na}_{2} \mathrm{PO}_{4} /$ $12 \mathrm{H}_{2} \mathrm{O}, 5.1 \mathrm{mg}$ of $\mathrm{NH}_{4} \mathrm{Cl}, 67.5 \mathrm{mg}$ of $\mathrm{MgSO}_{4} / 7 \mathrm{H}_{2} \mathrm{O}, 82.5$ $\mathrm{mg}$ of $\mathrm{CaCl}_{2}$ and $0.75 \mathrm{mg}$ of $\mathrm{FeCl}_{3} / 6 \mathrm{H}_{2} \mathrm{O}$ per liter. The $\mathrm{pH}$ of the medium was adjusted to 7.2. Incubation was carried out at $25^{\circ} \mathrm{C}$ under shaking. When growth was visible, the enrichment culture was streaked on agar plate containing the basal medium.

\section{Chemicals}

Dodecyltrimethylammonium bromide was purchased from Tokyo Kasei Kogyo Co., Ltd., Japan. Other reagents were of the highest purity available.

\section{Analytical methods}

${ }^{1} \mathrm{H}$ nuclear magnetic resonance spectrometry $\left({ }^{1} \mathrm{H}-\mathrm{NMR}\right)$, Ion chromatography and dissolved organic carbon analysis were used for the quantification and identification of DTAB and its degradation products.

${ }^{1} \mathrm{H}-\mathrm{NMR}$ analysis: The cultured medium in each flask was filtrated through a $\mathrm{GF} / \mathrm{C}$ filter and evaporated to dryness. The residue was dissolved in $\mathrm{d}_{4}$-methanol, passed through a $0.45 \mu \mathrm{m}$ filter, and again evaporated to dryness. This was followed by the addition of $\mathrm{D}_{2} \mathrm{O}$ containing disodium fumarate as an internal standard. The conditions for this analysis were as follows: frequency, $270 \mathrm{MHz}$; temperature, $40^{\circ} \mathrm{C}$; frequency range, $6000 \mathrm{~Hz}$; scan, 128 times; apparatus, JMN-EX270 (JEOL, Japan).

Ion chromatography analysis: The cultured medium in each flask was analyzed by ion chromatography after filtration. The conditions for ion chromatography were as follows: separation column, TSKgel IC-Cation $(4.6 \mathrm{~mm}$ i.d. $\times 50 \mathrm{~mm}$, TOSOH, Japan); mobile phase, $2 \mathrm{mM} \mathrm{HNO}_{3}$, $1.2 \mathrm{~mL} / \mathrm{min}$; temperature, $40^{\circ} \mathrm{C}$; apparatus, IC 7000 ion chromatographic analyzer (Yokogawa analytical systems, Japan).

DOC analysis: The concentration of dissolved organic carbon (DOC) in the filtrated culture medium was deter- mined with a TOC-5000 total organic carbon analyzer (Shimadzu Corp., Japan).

Biological oxygen demand (BOD) was determined using an automatic oxygen consumption measuring apparatus (Courometer, Okura Electric Co., Ltd., Japan) according to OECD modified MITI test (I) (1981). The volume of basal culture volume was $300 \mathrm{~mL}$ and the concentration of DTAB was $30 \mathrm{mg} / \mathrm{L}$.

\section{Results}

\section{Isolation and identification of DTAB-degrading bacteria}

Two strains of bacteria were isolated from the enrichment culture of the activated sludge. They were identified as Pseudomonas fluorescens according to Bergey's manual of Determinative Bacteriology (1974), based on morphological and physiological characteristics as well as electronmicroscopic findings (Table 1, respectively). These strains were called Ps. fluorescens F2 and Ps. fluorescens F7.

\section{Degradation activities of Ps. fluorescens F7 and F2}

The DTAB degradation activity of the isolates was represented as a ratio of the biochemical oxygen demand (BOD) to the theoretical oxygen demand (ThOD). Figure 1 shows the BOD degradability curve of F7, F2 and the activated sludge obtained from a WWTP. DTAB degradation by $P$. fluorescens F7 and F2 started within 48 hours, and the BOD degradability was $60 \%$ within a week. The two strains as well as the activated sludge had high-degrading activity.

\section{Degradation products of DTAB treated with}

Ps. fluorescens $F 7$ and $F 2$

DTAB and its degradation products in the cultured medium were identified and determined by ${ }^{1} \mathrm{H}-\mathrm{NMR}$ and ion chromatography. In the case of F7, trimethylamine (TMA) and dimethylamine (DMA) were detected. Figure 2 shows the time course changes in the concentration of DTAB and its intermediates during treatment with Ps. fluorescens F7. After 7 days of culture, TMA was initially detected and after 8 days dimethylammonium (DMA) was detected. After 9 days, the sum of molar concentrations of DTAB and its intermediates accounted for $32 \%$ of the initial concentration of DTAB.

From these results, we propose that the pathway of biodegradation of DTAB by Ps. fluorescens F7 is as follows: DTAB is initially degraded to TMA through an N-C bond cleavage (N-dealkylation); then, TMA is degraded to DMA by another N-C bond cleavage (N-demethylation) (Fig. 3). 
Table 1. Morphological and physiological characteristics of Pseudomonas fluorescens F2 and F7.

\begin{tabular}{|c|c|c|}
\hline & $\mathrm{F} 2$ & F7 \\
\hline Shape & $\operatorname{rod}$ & $\operatorname{rod}$ \\
\hline Gram reaction & - & - \\
\hline Spore & - & - \\
\hline Mortility & + & + \\
\hline Flagella & $\begin{array}{c}\text { Polar } \\
\text { (multitrichous) }\end{array}$ & $\begin{array}{c}\text { Polar } \\
\text { (multitrichous) }\end{array}$ \\
\hline Oxidase & + & + \\
\hline Catalase & + & + \\
\hline O-F test & $\mathrm{O}$ & $\mathrm{O}$ \\
\hline Color of colony & NP & NP \\
\hline Fluorescent pigments & + & + \\
\hline Water soluble pigments & + & + \\
\hline Accumulation of PHB & - & - \\
\hline Growth at $41^{\circ} \mathrm{C}$ & - & - \\
\hline Levan formation from sucrose & - & + \\
\hline Arginine dihydrolase & + & + \\
\hline Denitrification & + & + \\
\hline Nitrate reduction & + & + \\
\hline Gelatin liquefaction & + & - \\
\hline Starch hydrolysis & - & - \\
\hline Lecithinase & + & - \\
\hline Lipase (Tween 80 hydrolysis) & + & - \\
\hline Quinones & Q-9 & Q-9 \\
\hline DNA base ratio $\mathrm{G}+\mathrm{C}$, moles $\%$ & 63 & 62 \\
\hline \multicolumn{3}{|l|}{ Carbon sources for growth: } \\
\hline Glucose & + & + \\
\hline Trehalose & + & + \\
\hline 2-Ketoguluconate & + & + \\
\hline Inositol & - & + \\
\hline Geraniol & + & - \\
\hline L-Varine & + & + \\
\hline$\beta$-Alanine & + & + \\
\hline DL-Arginine & + & + \\
\hline L-Tartaric acid & - & - \\
\hline Ethanol & + & + \\
\hline D-Alanine & + & + \\
\hline Sucrose & - & + \\
\hline Mannitol & - & + \\
\hline Sorbitol & - & + \\
\hline Rhamnose & - & - \\
\hline
\end{tabular}

Although we did not detect any other product by the chemical analyses performed, it is probable that fatty acids, aliphatic aldehyde and/or alcohol were also produced but were immediately assimilated by this bacterium.

Figure 4 shows the time course changes in the concentration of DTAB and its intermediate by another DTAB de-

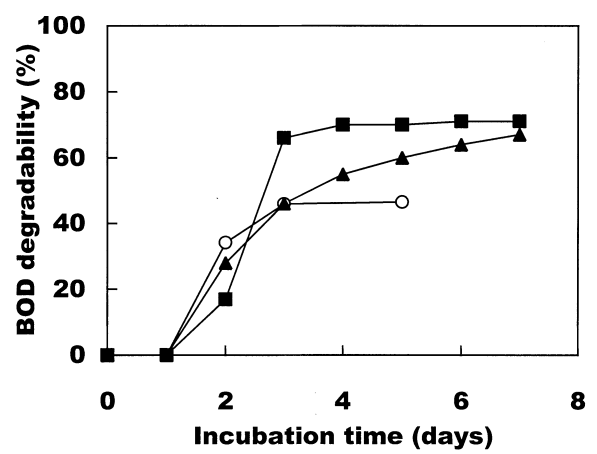

Fig. 1. BOD degradability curves of DTAB by isolated bacteria and activated sludge. Symbols are as follows: $\boldsymbol{\Delta}$, Pseudomonas fluorescens F2; $\mathbf{\square}$, Pseudomonas fluorescens F7; and $\bigcirc$, activated sludge.

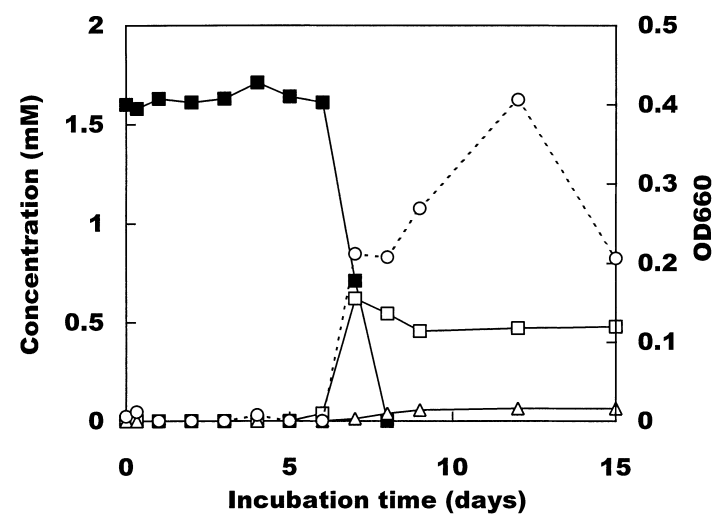

Fig. 2. Time course of DTAB degradation by Pseudomonas fluorescens $\mathrm{F} 7 . \mathbf{\square}$, DTAB; $\square$, TMA; $\triangle$, DMA; and $\bigcirc, \mathrm{OD}_{660}$.

grading bacteria $P$ s. fluorescens F2. This strain stoichiometrically produced TMA without further N-demethylation. DTAB was degraded by this bacterium to TMA as the terminal product, but in the environment TMA might be subjected to N-demethylation by other bacteria, for instance the Ps. fluorescens F7 strain described above (Fig. 3).

\section{Effects of co-existing chemicals on DTAB degradation by} Ps. fluorescens F7

The effects of co-existing chemicals on DTAB degradation caused by Ps. fluorescens F7 were evaluated. The co-existing chemicals used were dodecanal and dodecanoate as predicted intermediates of DTAB, yeast extracts and glucose which are generally used in the culture of bacteria, sodium dodecyl sulfates (SDS) and linear alkylbenzene sulfonates (LAS) of anionic surfactant.

Dodecanoate, SDS and LAS remarkably enhanced the degradation of DTAB by Ps. fluorescens F7 (Fig. 5). In the 


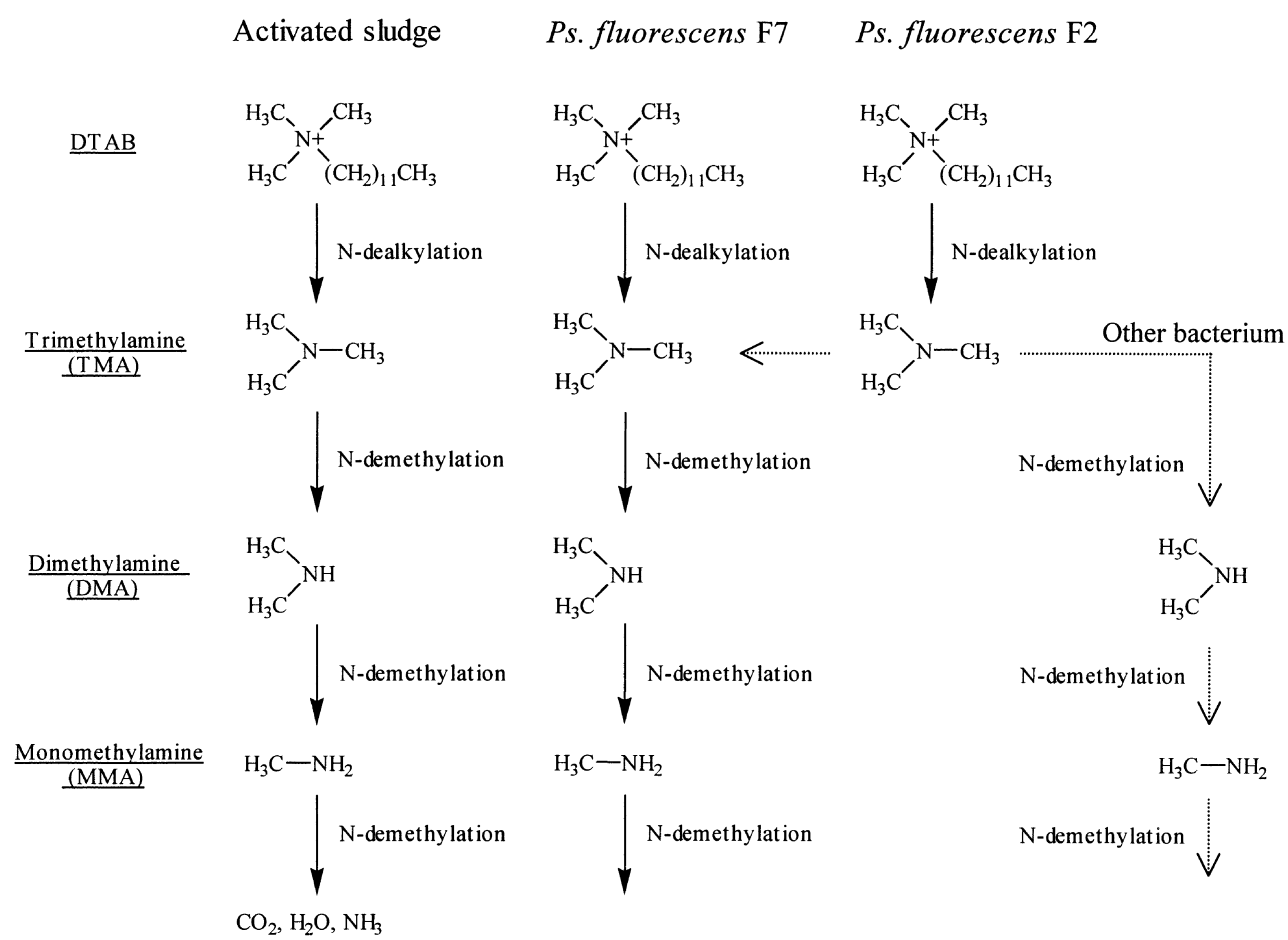

Fig. 3. Proposed degradation pathway of DTAB by Pseudomonas fluorescens F2 and F7.

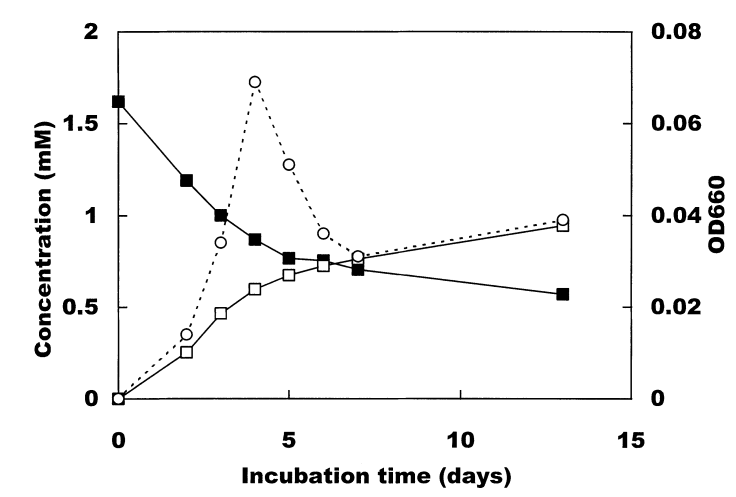

Fig. 4. Time course of DTAB degradation in Pseudomonas fluorescens F2. $\square$, DTAB; $\square$, TMA; and $\bigcirc, \mathrm{OD}_{660}$.

presence of dodecanoate or SDS, DTAB disappeared completely within 2 days under the incubation conditions of this study. All of these effective co-existing chemicals were anionic compounds.

Anionic compounds may accelerate the degradation of DTAB, because these chemicals form an ionic pair complex with DTAB, of cationic compound, reducing thereby its antimicrobial action. It is interesting to note that dodecanoate, one of the predicted intermediates, accelerates the degradation and becomes the sole source of carbon and energy for

\section{Ps. fluorescens F7.}

In contrast, DTAB degradation was not affected by the presence of dodecanal or yeast extracts, and glucose delayed the degradation of DTAB.

Effect of pre-incubation with DTAB on degradation by Ps. fluorescens $F 7$

Ps. fluorescens F7 pre-incubated in nutrient broth or basal medium was inoculated in basal medium. We assumed that if DTAB degradation by Ps. fluorescens F7 depended on an inducible enzyme, it affected the rate of DTAB. As shown in Figure 6, no difference in the rate of DTAB degradation between nutrient broth or basal medium for pre-incubation was observed, suggesting no involvement of inducible enzyme.

\section{Discussion}

Ps. fluorescens F7 and F2 isolated from activated sludge of a WWTP degraded DTAB to TMA by N-dealkylation, as the first step of the degradation process. This process was similar to that for alkyl (C10-C18) in activated sludge of a WWTP, as reported by Nishiyama et al. (1995). The BOD degradability of DTAB was also relatively high and similar to that of the activated sludge. These results suggested that 

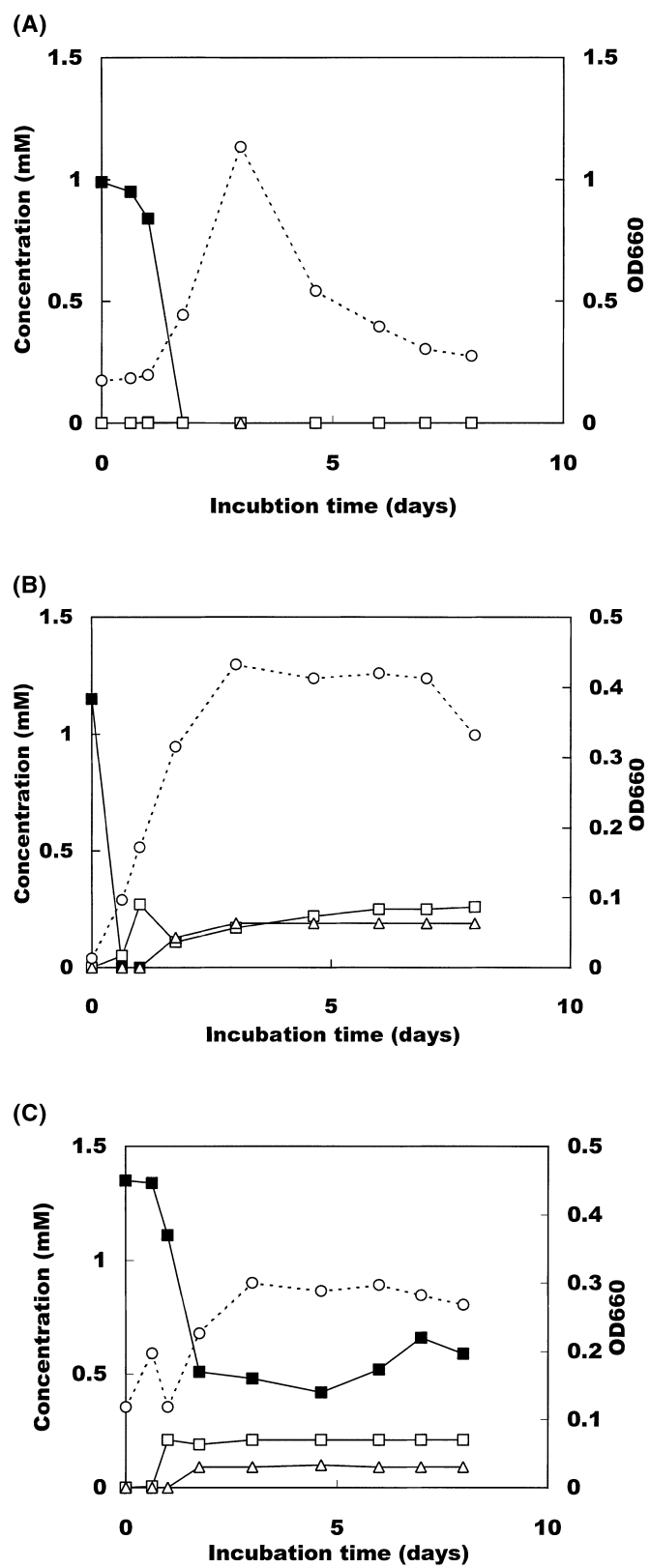

Fig. 5. Effects of dodecanoate (A), SDS (B) and LAS (C) on the degradation of DTAB. $\mathbf{\square}$, DTAB; $\square$, TMA; $\triangle$, DMA; and $\bigcirc$, $\mathrm{OD}_{660}$.

Ps. fluorescens F7 and F2 played an important role in DTAB degradation by activated sludge of a WWTP.

As illustrated in Figure 3, there was a difference in the degradation of DTAB between Ps. fluorescens F7 and F2. The TMA intermediate was degraded by Ps. fluorescens F7, but not by Ps. fluorescens F2. van Ginkel et al. (1992) have reported that Pseudomonas $s p$. isolated from activated sludge produced the final degradation product TMA from
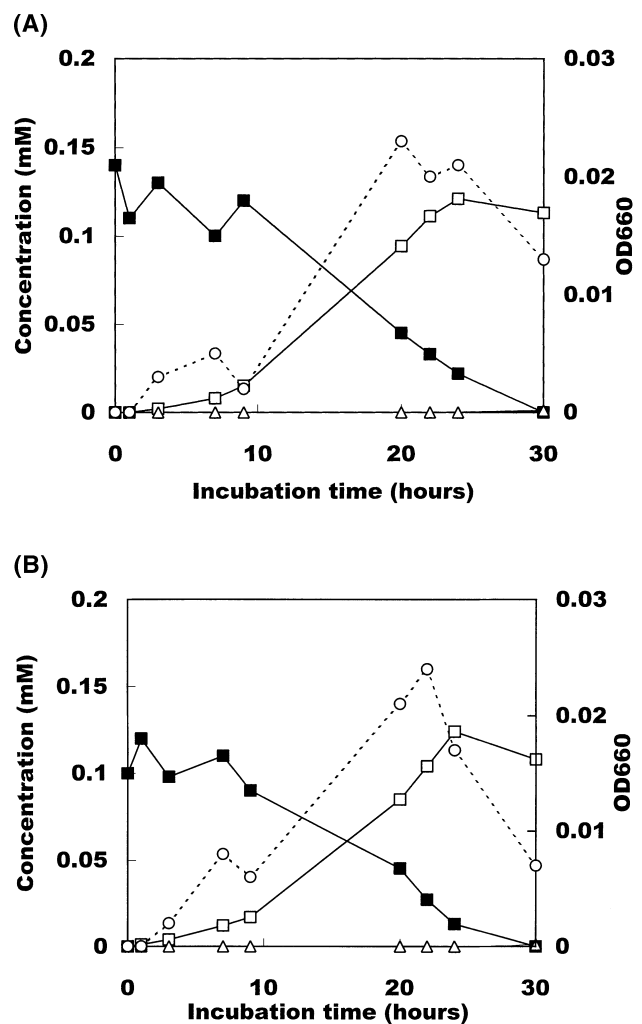

Fig. 6. Effect of DTAB degradation by pre-incubation, in the basal medium (A), nutrient broth (B). $\square$, DTAB; $\square$, TMA; $\triangle$, DMA; and $\bigcirc, \mathrm{OD}_{660}$.

hexadecyltrimethylammonium chloride. In our study, Ps. fluorescens $\mathrm{F} 2$ also produced TMA from DTAB. Although these microorganisms may play a role in the aquatic environment, mineralization of DTAB was not completely achieved. Consequently, for complete mineralization these microorganisms need the help of other microorganisms, which can degrade TMA.

On the other hand, Ps. fluorescens F7 produced TMA from DTAB through a N-dealkylation process, and then TMA was transformed to DMA. It appeared that the microorganisms, which ultimately degraded QACs having long alkyl chains alone as Ps. fluorescens F7 has the potential for, complete the mineralization without any help for other microorganisms. A kind of microorganism able to completely degrade QACs by itself, might be useful for the biological treatment of wastewater containing QACs.

The acceleration of DTAB degradation in the presence of dodecanoate, LAS or SDS, implies a relationship between DTAB and anionic compounds in the medium. Nishihara et al. (1998) proposed that sodium laurate depresses the biocidal activity of didecyldimethylammonium chloride 
(DDAC) against Staphilococcus aureus, because sodium laurate can form a less active complex with DDAC by both ionic and hydrophobic interactions. We thought that Ps. fluorescens $\mathrm{F} 7$ reduced DTAB degradation in medium containing $500 \mathrm{mg} / \mathrm{L}$ of DTAB without co-existing anionic chemicals, because the minimum inhibition concentration of DTAB was $125 \mathrm{mg} / \mathrm{L}$ for this microorganism. Masuda et al. (1976) showed that the biodegradability of QACs was stimulated by the addition of SDS to the medium. These findings suggested the DTAB degradation by Ps. fluorescens $\mathrm{F} 7$ might be accelerated by the formation of an $\mathrm{DTAB} /$ anionic co-existing chemical complex. Moreover, dodecanoate, one of the anionic co-existing chemicals tested, accelerates DTAB degradation and become the carbon source and energy source in the growth of Ps. fluorescens F7.

If the N-dealkylation of DTAB by Ps. fluorescens F7 depended on an inducible enzyme, DTAB N-dealkylation should be affected by the presence of DTAB in the pre-incubation medium. To determine the effect of the pre-incubation medium, the incubation was done in medium containing DTAB or nutrient broth. As shown in Figure 6, no effect was observed on the growth of Ps. fluorescens F7 pre-incubated in basal medium or nutrient broth. Although we cannot decide without investigating the enzyme level, this result suggested that N-dealkylation of DTAB did not depend on an inducible enzyme.

\section{Acknowledgements}

We thank our colleague Mr. M. Hanada from Kao Corporation, for technical support.

\section{References}

1) Bergey's manual of Determinative Bacteriology, 8th edition. 1974. Williams \& Wilkins, Baltimore.

2) Dean-Raymond, D. and M. Alexander. 1977. Bacterial metabolism of quaternary ammonium compounds. App. Environ. Microbiol. 33: 1037-1041.

3) Kakiichi, N. 1993. Toxicity and evaluation of disinfectants on activated sludge and protozoa, J. Antibact. Antifungal Agents 21: $347-356$.

4) Kikuchi, M., E. Kashiwazaki, S. Higashiyama, K. Kumon, T. Takada, K. Abe, Y. Sakagami and H. Yokoyama. 1996. Bactericidal activities of four disinfectants and didecyldimethylammonium chloride against MRSA strains isolated in a hospital. J. Antibact. Antifungal Agents 24: 391-396.

5) Larson, R.J. and R.D. Vashon. 1983. Adsortpion and biodegradation of cationic surfactants in laboratory and environmental systems, Dev. Ind. Microbiol. 24: 425-434.

6) Masuda, F., S. Machida and M. Kanno. 1976. Studies on the biodegradability of some cationic surfactants, p. 129-138, In Proceedings of the $7^{\text {th }}$ international congress on surface active agents, Moscow, USSR.

7) Nishihara, T., M. Yano, K. Kato and A. Takasaki. 1998. Neutralizing effect of sodium laurate on the bacteriocidal action of a quaternary ammonium disinfectant against Staphilococcus aureus, Biocontrol Science 3: 1-5.

8) Nishihara, T., T. Okamoto and N. Nishiyama. 2000. Biodegradation of didecyldimethylammonium chloride by Pseudomonas fluorescens TN4 isolated from activated sludge. J. Appl. Microbiol. 88: 641-647.

9) Nishiyama, N., Y. Toshima and Y. Ikeda. 1995. Biodegradation of alkyltrimethylammonium salts in activated sludge. Chemosphere 30: 593-603.

10) OECD. 1981. Ready biodegradability, Modified MITI test (I). In OECD Guidelines for Testing of Chemicals, 301C. OECD, Paris.

11) Stephans, J., R. Kinger and J. Kettering. 1994. In vitro comparison of the effectiveness of three surface disinfectants. J. Calif. Dent. Assoc. 22: 40-46.

12) van Ginkel, C.G., J.B. van Dijl and A.G.M. Kroon. 1992. Metabolism of hexadecyltrimethylammonium chloride in Pseudomonas strain B1. Appl. Environ. Microbiol. 58: 3083-3087. 